\title{
State Observer Synchronization Used in the Three-Dimensional Duffing System
}

\author{
Jian-Qun Han, ${ }^{1}$ Yue-Chao Ma, ${ }^{2}$ and Hong Sun ${ }^{3}$ \\ ${ }^{1}$ College of Engineering, Bohai University, Jinzhou 121013, China \\ ${ }^{2}$ Department of Electronic Science and Technology, Harbin Institute of Technology, Harbin 150001, China \\ ${ }^{3}$ Higher Vocational College, Bohai University, Jinzhou 121013, China \\ Correspondence should be addressed to Jian-Qun Han; hanjianqun@126.com
}

Received 20 February 2014; Accepted 10 March 2014; Published 3 April 2014

Academic Editor: Shen Yin

Copyright (C) 2014 Jian-Qun Han et al. This is an open access article distributed under the Creative Commons Attribution License, which permits unrestricted use, distribution, and reproduction in any medium, provided the original work is properly cited.

\begin{abstract}
Synchronization of chaotic systems has attracted extensive concern in the past few years. In this study, we investigate a new structure of Duffing system by the variable decomposition method. Then, we analyze the state observer synchronization based on the new Duffing system. It is proved theoretically that the designed observer can keep synchronization with Duffing chaotic system in transmitter. The design is presented reasonably with the conditional Lyapunov exponents, and its effectiveness is clearly shown in simulation results.
\end{abstract}

\section{Introduction}

Duffing equations are well-known nonlinear equations of applied science which are used as a powerful tool to discuss some important practical phenomena such as periodic orbit extraction, nonuniformity caused by an infinite domain, and nonlinear mechanical oscillators.

Synchronization is a very common phenomenon of nonlinear dynamic system. It shows that two moving objects can make their states converge through the medium strong or weak coupling. Since Pecora and Carroll [1] put forward the principle of chaotic synchronization, synchronization of chaotic systems has become one of research hotspots in the field of nonlinear dynamics. Chaos synchronization has been widely applied to many fields such as biology, chemistry, medicine, electronics, information science, and secure communication. So far, there are various synchronous types [2] including complete synchronization, lag synchronization, anticipated synchronization, and generalized synchronization. Furthermore, many synchronous implementation methods have been studied which included drive-response synchronization, adaptive synchronization [3], impulsive synchronization [4], observer synchronization [5], and the sliding mode control [6]. The above methods are commonly used in data-driven fault diagnosis [7-11].

For obtaining synchronization, both the structure and the parameters of target system are often supposed to be available, while the only unknown is the initial condition. As the model of a system is commonly imprecise, obviously, it is not necessarily a realistic case. Therefore, the synchronization or autosynchronization of chaotic systems with unknown parameters has been widely studied in recent years. The synchronization of Lorenz system, one of the most popular chaotic systems, is stressed, and the conditions of synchronization have been studied by various researchers [12-20]. The main strategy is based on the adaptive feedback control. It is illustrated that synchronization is achievable, while the global convergence is guaranteed by the Lyapunov convergence theorem [12-16]. However, the proof of global convergence poses a restrictive form of the system. Thus, the unknown parameters can only appear when the system has to be transformed into some particular forms, such as stateaffine form, or in the dynamical equation of the observable state.

Due to easy engineering implementation, the observer synchronization method has gained extensive attention. 
Observer synchronization has simple structure, fast synchronous speed, and high precision. The method can be widely used in communications and information processing.

At present, the study of Duffing equation is in the form of two-dimensional state equation [21-24]. Three-dimensional Duffing state equation is presented in this paper. We combined the state observer with chaotic synchronization and proved the rationality of the nonlinear synchronous observer.

This paper is organized as follows. In Section 2, threedimensional Duffing state equation is presented. The phase diagrams of new Duffing system are also given. In Section 3, the basic idea of the adaptive observer design is described in detail. With supportive simulation results, the conditional Lyapunov exponents of the system are then studied in Section 4. Finally, conclusions are drawn, respectively, in Section 5 .

\section{Three-Dimensional Duffing System}

There are many problems in engineering, such as packaging systems based on displacement excitation of nonlinear vibrations, pressure sensors nonlinear vibration. They can be simplified into a forced Duffing equation with cubic nonlinearity, governed by

$$
\ddot{x}(t)+\mu \dot{x}(t)+\omega_{0}^{2} x(t)+\varepsilon x(t)^{3}=F \cos \Omega t,
$$

where $x(t)$ is an unknown real function; $\mu, \omega_{0}, \varepsilon, F, \Omega$ are known physical parameters; and the dot represents differentiation with respect to $t$.

Definition 1. The particular form of Duffing system related to system (1) is described as

$$
\begin{aligned}
& \dot{x}_{1}=x_{2}, \\
& \dot{x}_{2}=x_{1}-x_{1}^{3}-k x_{2}+r \cos \omega t,
\end{aligned}
$$

where $r$ and $\omega$ are the amplitude and frequency of driving force, respectively, $k$ is damping ratio, and $x_{1}-x_{1}^{3}$ is the nonlinear restoring force.

When given the system initial state $x_{1}(0)=0, x_{2}(0)=$ 1 in (2), two-dimensional Duffing system under sinusoidal signal drive produces the chaotic phase diagram as shown in Figure 1.

Definition 2. Given a constant $b$, system (2) can be converted into state equation

$$
\begin{aligned}
& \dot{x}_{1}=x_{2}, \\
& \dot{x}_{2}=-k x_{2}-x_{3}+b+r \cos \omega t, \\
& \dot{x}_{3}=3 x_{1}^{2} x_{2}-x_{2} .
\end{aligned}
$$

Proof. Choose the following differential equation:

$$
\dot{x}_{3}=3 x_{1}^{2} x_{2}-x_{2} \text {. }
$$

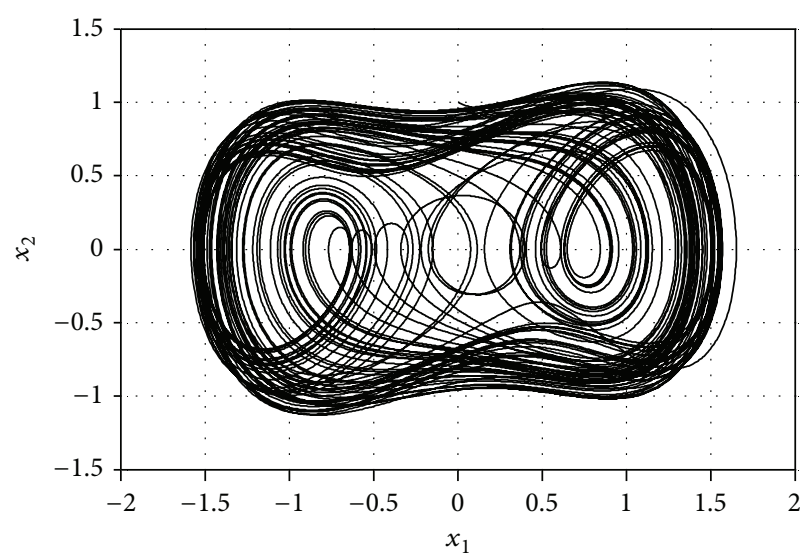

FIgUre 1: Two-dimensional Duffing chaotic system phase diagram with $x_{1}(0)=0$ and $x_{2}(0)=1$.

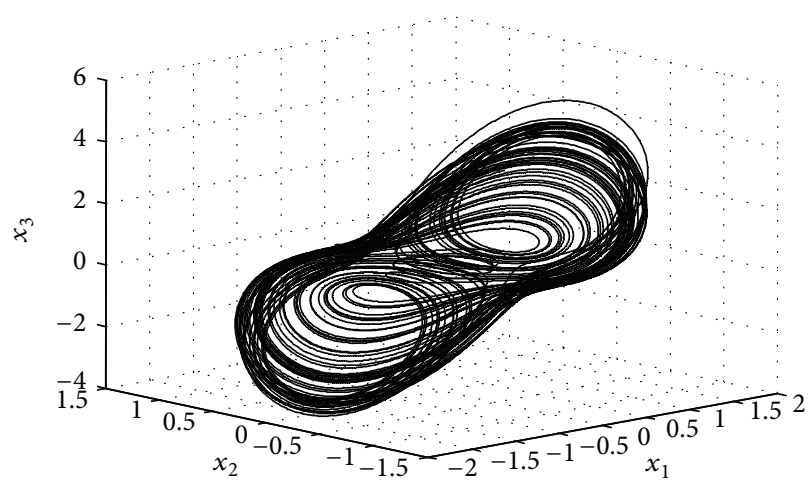

FIGURE 2: Three-dimensional Duffing chaotic system phase diagram with $x_{1}(0)=0, x_{2}(0)=1, x_{3}(0)=0$, and $b=0$.

Intergrating $\dot{x}_{3}$ taken in (4) for system (2) yields

$$
x_{3}=x_{1}^{3}-x_{1}+c,
$$

where $c$ is undetermined constant, and the value can be determined by the system initial state.

Remark 3. From the above analysis, it follows that $c$ and $b$ are unbounded, but $b$ is used to counteract the effects of $c$. When given $x_{1}(0)=0$ and $x_{3}(0)=0$ in the system (3), $c=0$ and $b=0$ hold. The three-dimensional Duffing chaotic dynamic system (3) is the same as system (2). Under the general conditions, system (2) can be modified to system (3) by adjusting variable $b$ to offset the impact of variable $c$.

When given $x_{1}(0)=0, x_{2}(0)=1, x_{3}(0)=0$, and $b=0$ in (3), three-dimensional Duffing system produces the chaotic phase diagram shown in Figure 2. Its projections on the xoy, yoz, and $\mathrm{xoz}$ coordinate plane are shown in Figures 3, 4, and 5. By comparing Figures 1 and 3, we can obtain the result that two-dimensional Duffing system and threedimensional Duffing system are consistent. Figures 4 and 5 can only produce in three-dimensional Duffing system.

The simulation results of equivalent condition are the premise of system initial states $x_{1}(0)=0$ and $x_{3}(0)=0$; otherwise the system status may not be chaotic. When given 


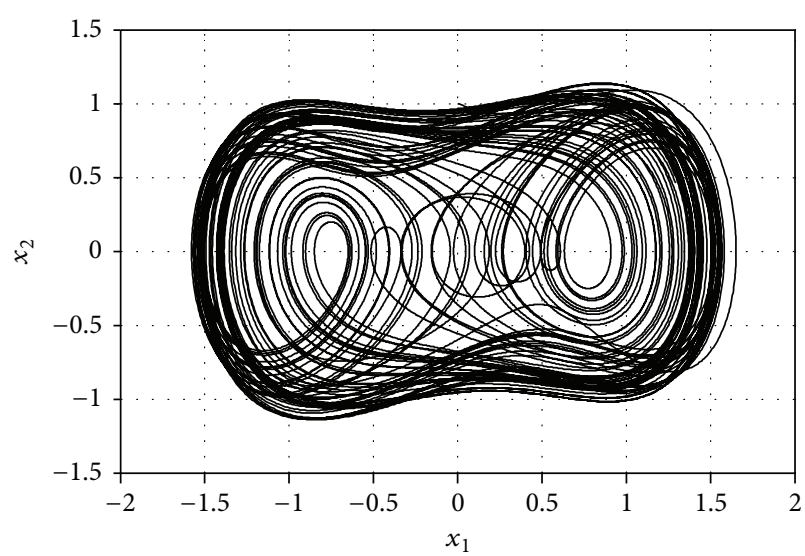

FIgURE 3: Three-dimensional Duffing chaotic system phase diagram in the xoy plane projection with $x_{1}(0)=0, x_{2}(0)=1, x_{3}(0)=0$, and $b=0$.

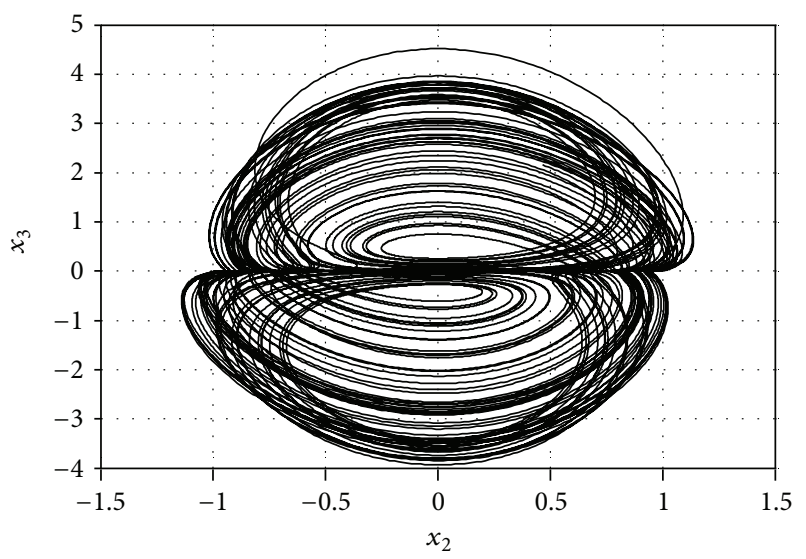

FIGURE 4: Three-dimensional Duffing chaotic system phase diagram in the yoz plane projection with $x_{1}(0)=0, x_{2}(0)=1, x_{3}(0)=0$, and $b=0$.

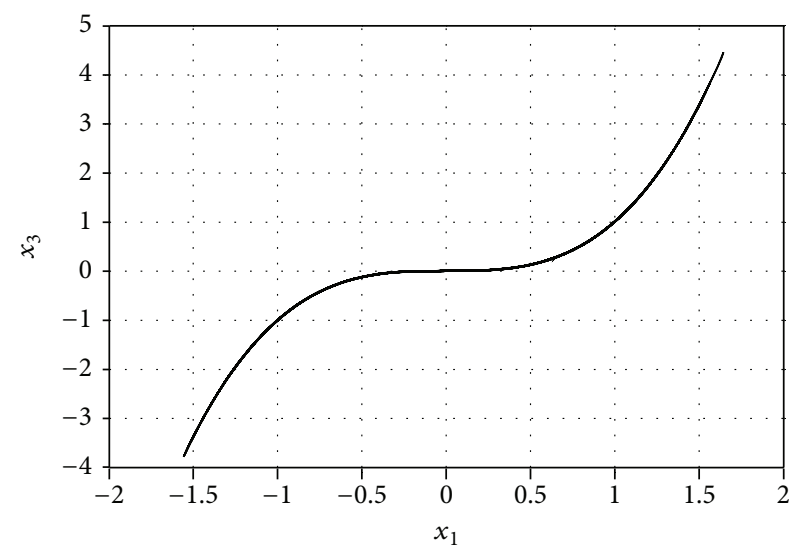

FIGURE 5: Three-dimensional Duffing chaotic system phase diagram in the xoz plane projection with $x_{1}(0)=0, x_{2}(0)=1, x_{3}(0)=0$, and $b=0$.

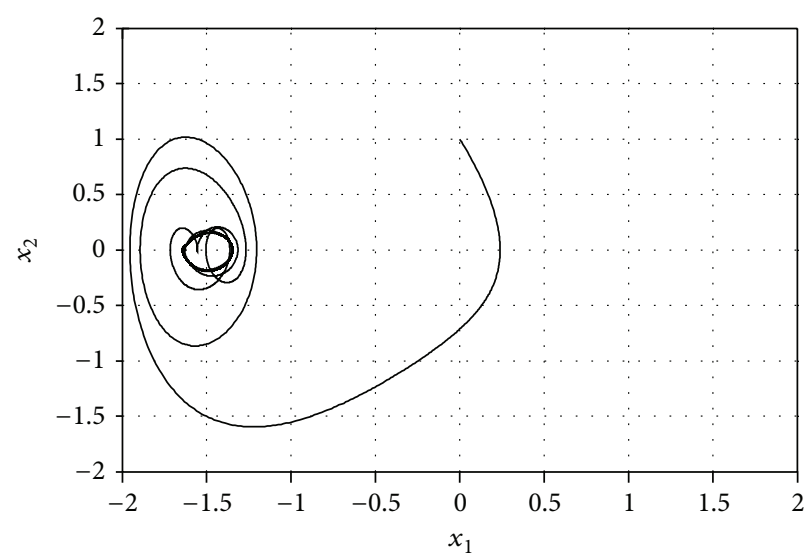

FIGURE 6: Three-dimensional Duffing chaotic system phase diagram in the xoy plane projection with $x_{1}(0)=0, x_{2}(0)=1, x_{3}(0)=2$, and $b=0$.

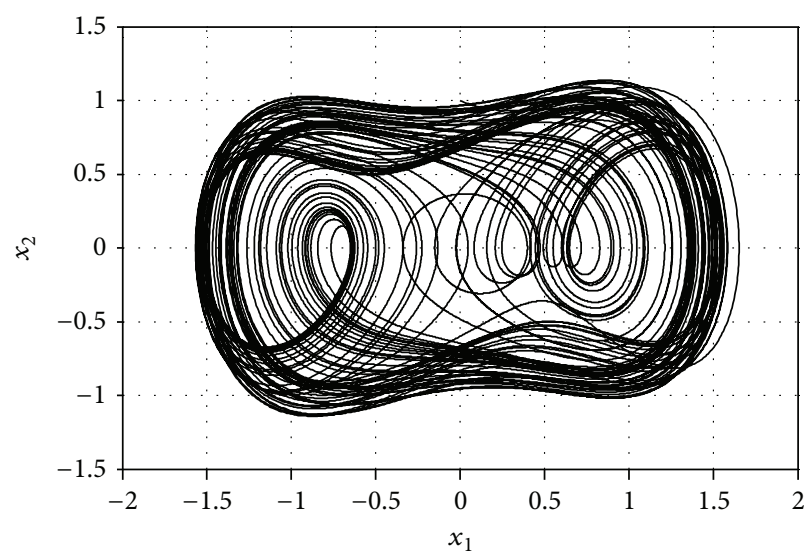

FIGURE 7: Three-dimensional Duffing chaotic system phase diagram in the xoy plane projection with $x_{1}(0)=0, x_{2}(0)=1, x_{3}(0)=2$, and $b=2$.

$x_{1}(0)=0, x_{2}(0)=1$, and $x_{3}(0)=2$, simulation result is shown in Figure 6 . The system state is not chaotic.

When it is assumed that $b=2$ in (3), the system phase diagram as shown in Figure 7 is chaotic. From the above analysis, results counteracting the effects of initial condition on system state and keeping the system chaotic can be obtained by adjusting the variable $b$.

\section{State Observer Synchronization}

Definition 4. Suppose that there exist

$$
\begin{gathered}
A=\left[\begin{array}{ccc}
0 & 1 & 0 \\
0 & -k & -1 \\
1 & 1 & -1
\end{array}\right], \quad B=\left[\begin{array}{lll}
1 & & \\
& 1 & \\
& & 1
\end{array}\right], \\
E=\left[\begin{array}{l}
0 \\
1 \\
0
\end{array}\right]
\end{gathered}
$$




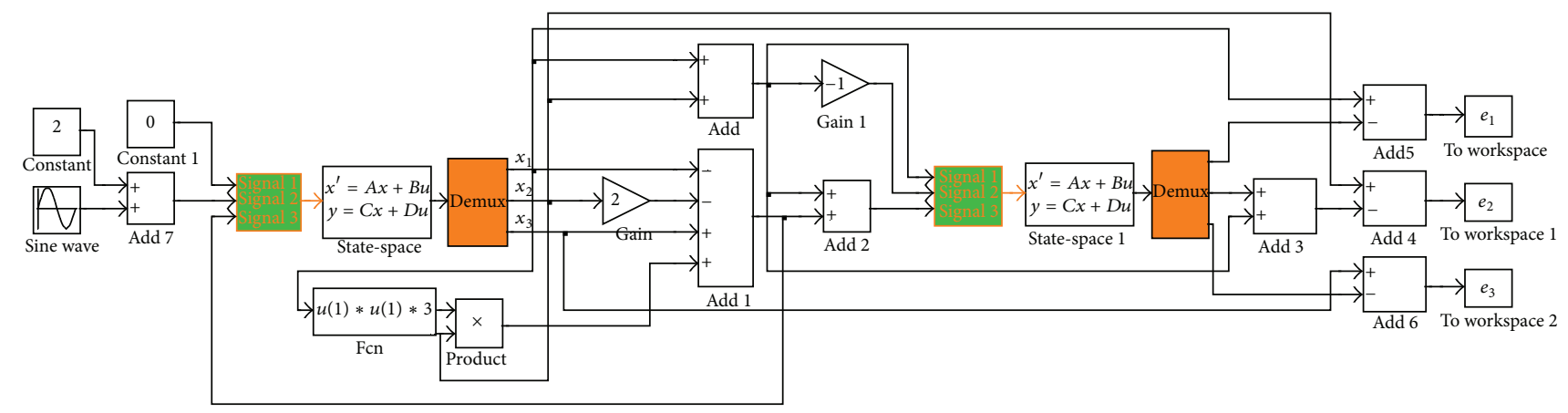

FIGURE 8: State observer synchronization simulation system used in three-dimensional Duffing system.

such that

$$
\begin{aligned}
\dot{x}= & {\left[\begin{array}{ccc}
0 & 1 & 0 \\
0 & -k & -1 \\
1 & 1 & -1
\end{array}\right] x+\left[\begin{array}{ccc}
1 & & \\
& 1 & \\
& & 1
\end{array}\right] } \\
& \times\left[\begin{array}{c}
0 \\
b \\
3 x_{1}^{2} x_{2}-x_{1}-2 x_{2}+x_{3}
\end{array}\right]+\left[\begin{array}{l}
0 \\
1 \\
0
\end{array}\right] r \cos \omega t .
\end{aligned}
$$

Proof. For (7), written in general form as follows:

$$
\begin{gathered}
\dot{x}=A x+B u+E w, \\
y=C^{T} x, \\
u=g(x),
\end{gathered}
$$

where $x, u$, and $w$ are, respectively, the system state variables, the nonlinear state feedback input, and system driver, $x \in R^{n}$, $u \in R^{k}$, and $w \in R^{m} ; y \in R^{p}$ is the output of sending system; coefficients are $n=3, k=3, m=1$, and $p=1$.

There exist $\operatorname{rank} E=m, \operatorname{rank} C^{T}=p$, and $\operatorname{rank} C^{T} E=m$ in three-dimensional Duffing system. Notice that $p \geq m$ here.

At the receiving system, when the nonlinear input feedback can be measured, system (8) for state observer can be defined in (9). Consider

$$
\begin{gathered}
\dot{z}=\widehat{G} z+\widehat{F} u+\widehat{J} y, \\
\widehat{x}=z-\widehat{H} y,
\end{gathered}
$$

where $z \in R^{n}$ and $\widehat{x} \in R^{n} ; \widehat{G}, \widehat{F}, \widehat{J}$, and $\widehat{H}$ are the unknown constant matrix.

Now define $e=\widehat{x}-x=z-\left(I_{n}+\widehat{H} C^{T}\right) x$ and $\widehat{N}=I_{n}+\widehat{H} C^{T}$. Then, we obtain the dynamic equation of the error system that

$$
\dot{e}=\widehat{G} e+\left(\widehat{J} C^{T}-\widehat{N} A+\widehat{G} \widehat{N}\right) x+(\widehat{F}-\widehat{N} B) u-\widehat{N} E w .
$$

If system (10) has

$$
\begin{gathered}
\left(I_{n}+\widehat{H} C^{T}\right) E=0, \\
\widehat{F}-\widehat{N} B=0, \\
\widehat{J} C^{T}-\widehat{N} A+\widehat{G} \widehat{N}=0,
\end{gathered}
$$

then it can be rewritten as

$$
\dot{e}=\widehat{G} e .
$$

Remark 5. If all eigenvalues of $\widehat{G}$ are less than zero, then $\widehat{x}$ will exponentially converge at $x$.

By $\widehat{J} C^{T}-\widehat{N} A+\widehat{G} \widehat{N}=0$, we can obtain

$$
\widehat{G}=\widehat{N} A-(\widehat{J}+\widehat{G} \widehat{H}) C^{T} .
$$

Let $\widehat{M}=\widehat{J}+\widehat{G} \widehat{H}$. Then, we can obtain

$$
\widehat{J}=\widehat{M}+\widehat{M} C^{T} \widehat{H}-\widehat{N} A \widehat{H},
$$

which leads to

$$
\dot{z}=\left(\widehat{N} A-\widehat{M} C^{T}\right) z+\widehat{F} u+\widehat{J} y .
$$

So the state observer design problem turns into finding the right $\widehat{H}$ and $\widehat{M}$. To make it meet $\left(I_{n}+\widehat{H} C^{T}\right) E=0$ and $\operatorname{eig}(\widehat{G})<0$.

From (15), we can obtain

$$
\widehat{G}=\widehat{N} A-\widehat{M} C^{T} .
$$

Remark 6. All eig $(\widehat{G})<0$, if $\exists$ for all $\widehat{M} \in R^{n \times p}$ and $B=\left\{x \mid x=\operatorname{eig}\left(\widehat{N} A-\widehat{M} C^{T}\right)\right.$, for all $\left.x \in R\right\}$. Namely, the eigenvalues of $\widehat{N} A-\widehat{M} C^{T}$ can be set arbitrarily if $\left(\widehat{N} A, C^{T}\right)$ can be completely observable.

Proof. By (11), we can get $\widehat{H} C^{T} E=-E$ and $\operatorname{rank} C^{T} E=m$. So we can obtain

$$
\widehat{H}=-E\left(C^{T} E\right)^{+}+Y\left(I_{p}+\left(C^{T} E\right)\left(C^{T} E\right)^{+}\right),
$$

where $Y \in R^{n \times p}, n, p \in R$; $Y$ is the right dimension of arbitrary matrix.

Consider the expression $\widehat{N}=I_{n}+\widehat{H} C^{T}$; we can obtain

$$
\widehat{N}=\left(I_{n}+Y C^{T}\right)\left(I_{n}-E\left(C^{T} E\right)^{+} C^{T}\right) .
$$




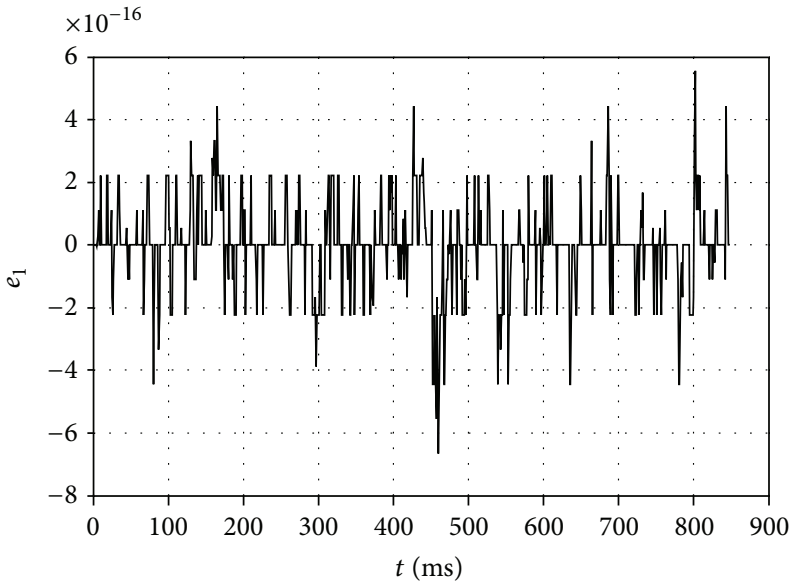

(a) The curve $e_{1}$ for $x_{1}-\widehat{x}_{1}$

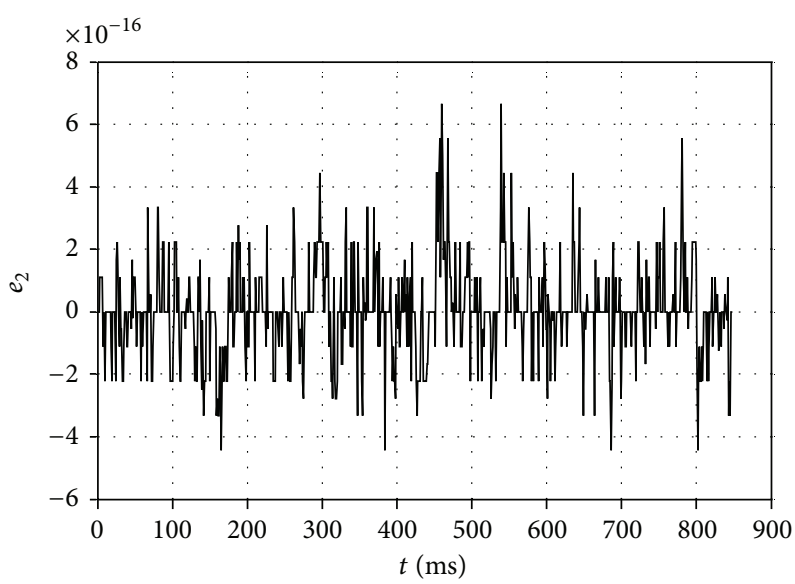

(b) The curve $e_{2}$ for $x_{2}-\widehat{x}_{2}$

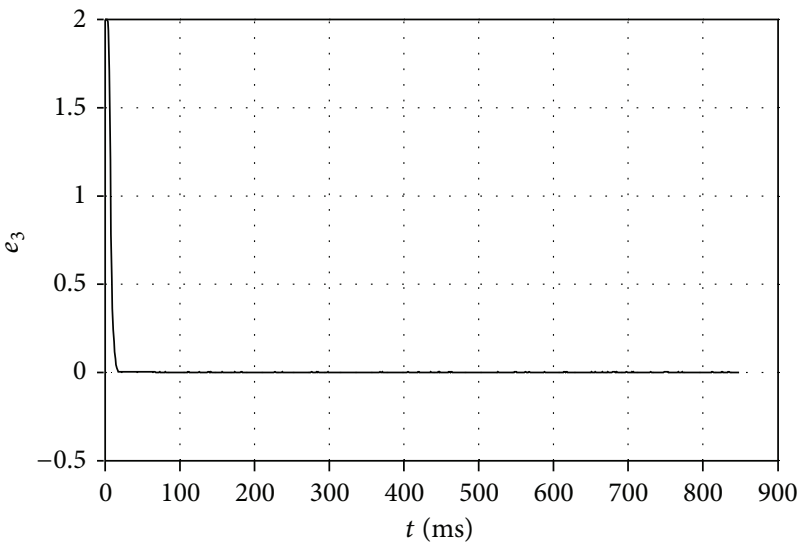

(c) The curve $e_{3}$ for $x_{3}-\widehat{x}_{3}$

Figure 9: The error curves driven by the outside periodic force $0.7 \cos t$ and the damping ratio fixed to 0.5 . The parameter $b$ can be modified. There is no interference.

Theorem 7. Assume $\operatorname{rank} \widehat{N}=n-m$ if $\left|I_{n}+Y C^{T}\right| \neq 0$ and $\left|I_{p}+C^{T} Y\right| \neq 0$. One can obtain

$$
\operatorname{rank}\left[\begin{array}{c}
C^{T} \\
C^{T} \widehat{N} A \\
\vdots \\
C^{T}(\widehat{N} A)^{n-1}
\end{array}\right]=n
$$

which yields that $\left(\widehat{N} A, C^{T}\right)$ can be completely observable and the eigenvalues of $\widehat{G}$ can be arbitrarily set.

By Theorem 7, we know that $\left|I_{p}+C^{T} Y\right| \neq 0$ if $\operatorname{rank} C=p$ and $Y=0$. Then, we have

$$
\widehat{N}=I_{n}-E\left(C^{T} E\right)^{+} C^{T}
$$

Remark 8. When $\widehat{N}=I_{n}-E\left(C^{T} E\right)^{+} C^{T}$, we can always find a suitable $\widehat{M}$, making eig $(\widehat{G})<0$. Namely, $\widehat{x} \rightarrow x$ for $\forall t \rightarrow$ $\infty$.
Now let

$$
\begin{gathered}
A=\left[\begin{array}{ccc}
0 & 1 & 0 \\
0 & -0.5 & -1 \\
1 & 1 & -1
\end{array}\right], \quad B=\left[\begin{array}{lll}
1 & \\
& 1 & \\
& & 1
\end{array}\right], \\
E=\left[\begin{array}{l}
0 \\
1 \\
0
\end{array}\right], \quad C=\left[\begin{array}{l}
1 \\
1 \\
0
\end{array}\right], \\
u(t)=\left[\begin{array}{c}
0 \\
b \\
3 x_{1}^{2} x_{2}-x_{1}-2 x_{2}+x_{3}
\end{array}\right], \\
w(t)=r \cos \omega t \quad \text { in }(7) .
\end{gathered}
$$

From the above analysis, we can obtain

$$
\begin{gathered}
\widehat{H}=-E\left(C^{T} E\right)^{+}=\left[\begin{array}{c}
0 \\
-1 \\
0
\end{array}\right], \\
\widehat{N}=I_{n}+\widehat{H} C^{T}=\left[\begin{array}{ccc}
1 & 0 & 0 \\
-1 & 0 & 0 \\
0 & 0 & 1
\end{array}\right]
\end{gathered}
$$


Let $\widehat{M}=\left[\begin{array}{l}1 \\ 1 \\ 1\end{array}\right]$. Then we obtain $\widehat{G}=\left[\begin{array}{ccc}-1 & 0 & 0 \\ -1 & -2 & 0 \\ 0 & 0 & -1\end{array}\right]$ and $\operatorname{eig}(\widehat{G})=(-2,-1,-1)$. All eigenvalues of $\widehat{G}$ are negative. Then $\widehat{x} \rightarrow x$ as $t \rightarrow \infty$. So we can obtain the receiving system:

$$
\begin{aligned}
& \dot{z}=\left[\begin{array}{ccc}
-1 & 0 & 0 \\
-1 & -2 & 0 \\
0 & 0 & -1
\end{array}\right] z+\left[\begin{array}{ccc}
1 & 0 & 0 \\
-1 & 0 & 0 \\
0 & 0 & 1
\end{array}\right] u(t)+\left[\begin{array}{c}
1 \\
-1 \\
1
\end{array}\right] y, \\
& \hat{x}=z-\left[\begin{array}{c}
0 \\
-1 \\
0
\end{array}\right] y .
\end{aligned}
$$

\section{Simulation and Analysis}

In this section, we evaluate the performance of state observer synchronization used in three-dimensional Duffing system. Under the condition of interference and no interference, we simulated transceiver system synchronization.

To discuss the synchronization performance of system, we assumed coefficient of transmitting system $k=0.5, b=2$, $r=0.7$, and $\omega=1$ in (7). The synchronous state observer is designed as a receiving system. The simulation system based on MATLAB is shown in Figure 8.

Under the condition of no interference, synchronization error is shown in Figure 9. There exist $e_{1}=x_{1}-\widehat{x}_{1}, e_{2}=$ $x_{2}-\widehat{x}_{2}$, and $e_{3}=x_{3}-\widehat{x}_{3}$. Error curves tend to zero. Simulation results show that transceiver system can keep the good synchronization performance.

When there is interference, we assume adding Gaussian white noise to $u(t)$ or $w(t)$ in (8). Assume noise power spectrum density $n_{0}=0.1$. Error curves tend to zero too. The simulation results are similar to the Figure 9 without interference.

\section{Conclusions}

The present work has studied a new three-dimensional chaotic synchronization of Duffing system. The chaotic system is different from the previous Duffing system. It contains three equations. The third equation describes nonlinear effect on the new Duffing system. This system redefined state variables, nonlinear feedback input, and driving force. Because the synchronization method based on observer does not need the initial state in the same basin of attraction, we prove observer synchronization in three-dimensional Duffing system. By adopting the method of simple linear observer, receiving system can successfully keep synchronization with transmitting chaotic system.

\section{Conflict of Interests}

The authors declare that there is no conflict of interests regarding the publication of this paper.

\section{Acknowledgment}

This work is supported by the National Natural Science Foundation of China under Grant no. 51277011.

\section{References}

[1] L. M. Pecora and T. L. Carroll, "Synchronization in chaotic systems," Physical Review Letters, vol. 64, no. 8, pp. 821-824, 1990.

[2] S. Boccaletti, J. Kurths, G. Osipov, D. L. Valladares, and C. S. Zhou, "The synchronization of chaotic systems," Physics Reports, vol. 366, no. 1-2, pp. 1-101, 2002.

[3] H. Salarieh and A. Alasty, "Adaptive chaos synchronization in Chua's systems with noisy parameters," Mathematics and Computers in Simulation, vol. 79, no. 3, pp. 233-241, 2008.

[4] C. Li, X. Liao, and R. Zhang, "Impulsive synchronization of nonlinear coupled chaotic systems," Physics Letters A, vol. 328, no. 1, pp. 47-50, 2004.

[5] G. Millerioux, F. Anstett, and G. Bloch, "Considering the attractor structure of chaotic maps for observer-based synchronization problems," Mathematics and Computers in Simulation, vol. 68, no. 1, pp. 67-85, 2005.

[6] M. Roopaei, B. Ranjbar Sahraei, and T.-C. Lin, "Adaptive sliding mode control in a novel class of chaotic systems," Communications in Nonlinear Science and Numerical Simulation, vol. 15, no. 12, pp. 4158-4170, 2010.

[7] S. Yin, H. Luo, and S. Ding, "Real-time implementation of faulttolerant control systems with performance optimization," IEEE Transactions on Industrial Electronics, vol. 61, no. 5, pp. 24022411, 2014.

[8] S. Yin, G. Wang, and H. Karimi, "Data-driven design of robust fault detection system for wind turbines," Mechatronics, 2013.

[9] S. Yin, S. X. Ding, A. H. A. Sari, and H. Hao, "Data-driven monitoring for stochastic systems and its application on batch process," International Journal of Systems Science, vol. 44, no. 7, pp. 1366-1376, 2013.

[10] S. Yin, S. Ding, A. Haghani, H. Hao, and P. Zhang, "A comparison study of basic data-driven fault diagnosis and process monitoring methods on the benchmark Tennessee Eastman process," Journal of Process Control, vol. 22, no. 9, pp. 1567-1581, 2012.

[11] S. Yin, X. Yang, and H. R. Karimi, "Data-driven adaptive observer for fault diagnosis," Mathematical Problems in Engineering, vol. 2012, Article ID 832836, 21 pages, 2012.

[12] D. Huang, "Synchronization-based estimation of all parameters of chaotic systems from time series," Physical Review E, vol. 69, no. 6, Article ID 067201, 4 pages, 2004.

[13] D. Huang, "Adaptive-feedback control algorithm," Physical Review E, vol. 73, no. 6, Article ID 066204, 8 pages, 2006.

[14] C. Tao, Y. Zhang, G. H. Du, and J. J. Jiang, "Estimating model parameters by chaos synchronization," Physical Review E, vol. 69, no. 3, Article ID 036204, 2004.

[15] S. Chen and J. Lü, "Parameters identification and synchronization of chaotic systems based upon adaptive control," Physics Letters A, vol. 299, no. 4, pp. 353-358, 2002.

[16] G. Besançon, J. de León-Morales, and O. Huerta-Guevara, "On adaptive observers for state affine systems," International Journal of Control, vol. 79, no. 6, pp. 581-591, 2006.

[17] U. Parlitz, "Estimating model parameters from time series by autosynchronization," Physical Review Letters, vol. 76, no. 8, pp. 1232-1235, 1996.

[18] U. Parlitz, L. Junge, and L. Kocarev, "Synchronization-based parameter estimation from time series," Physical Review E, vol. 54, no. 6, pp. 6253-6259, 1996. 
[19] A. Maybhate and R. E. Amritkar, "Use of synchronization and adaptive control in parameter estimation from a time series," Physical Review E, vol. 59, no. 1, pp. 284-293, 1999.

[20] A. Maybhate and R. E. Amritkar, "Dynamic algorithm for parameter estimation and its applications," Physical Review E, vol. 61, no. 6B, pp. 6461-6470, 2000.

[21] H. Carmen, Z. Q. Lang, and S. A. Billings, "A frequency domain analysis of the effects of nonlinear damping on the Duffing equation," Mechanical Systems and Signal Processing, vol. 45, pp. 49-67, 2014.

[22] Z. Cheng and J. Ren, "Harmonic and subharmonic solutions for superlinear damped Duffing equation," Nonlinear Analysis: Real World Applications, vol. 14, no. 2, pp. 1155-1170, 2013.

[23] M. Zapateiro, Y. Vidal, and L. Acho, "A secure communication scheme based on chaotic Duffing oscillators and frequency estimation for the transmission of binary-coded messages," Communications in Nonlinear Science and Numerical Simulation, vol. 19, no. 4, pp. 991-1003, 2014.

[24] M. U. Akhmet and M. O. Fen, "Chaotic period-doubling and OGY control for the forced Duffing equation," Communications in Nonlinear Science and Numerical Simulation, vol. 17, no. 4, pp. 1929-1946, 2012. 


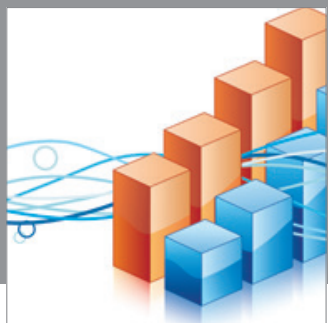

Advances in

Operations Research

mansans

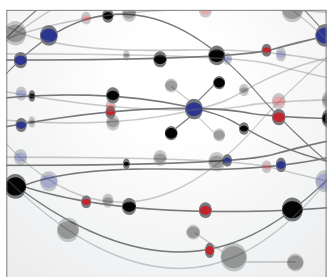

The Scientific World Journal
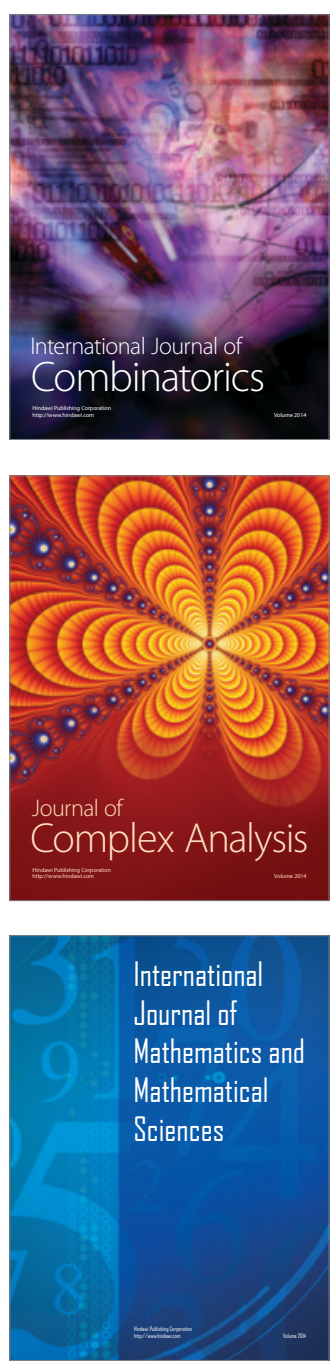
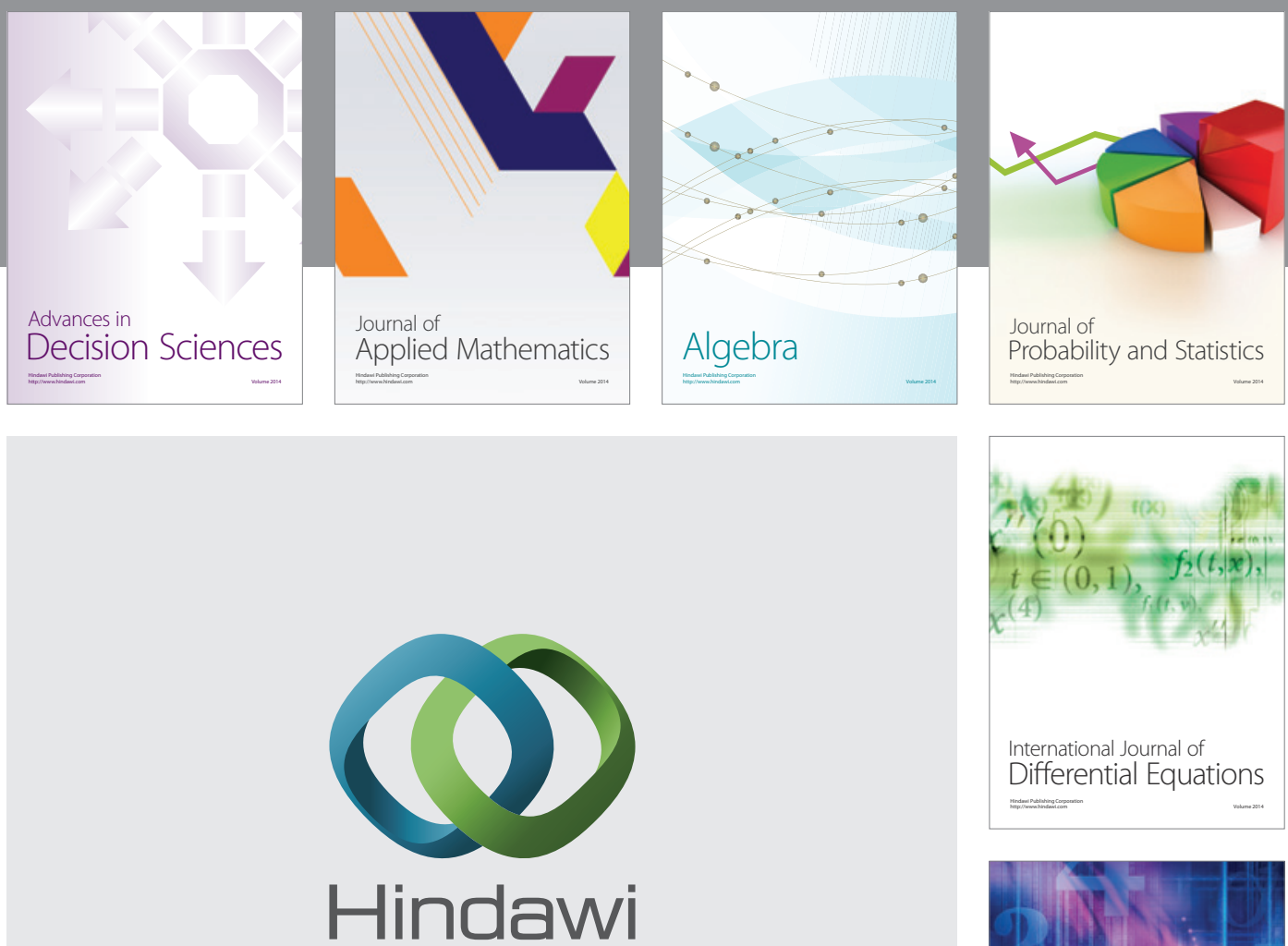

Submit your manuscripts at http://www.hindawi.com
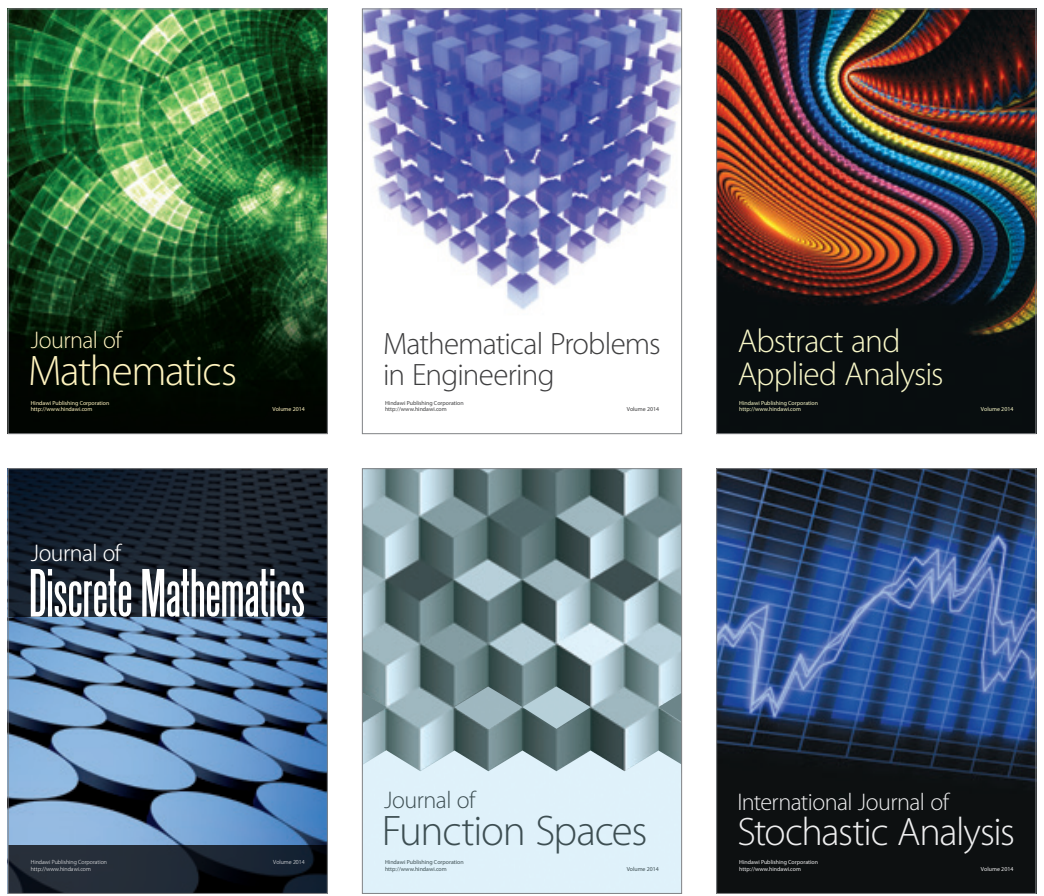

Journal of

Function Spaces

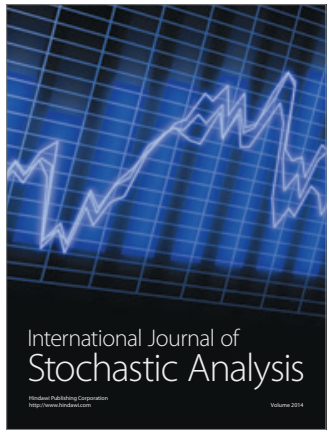

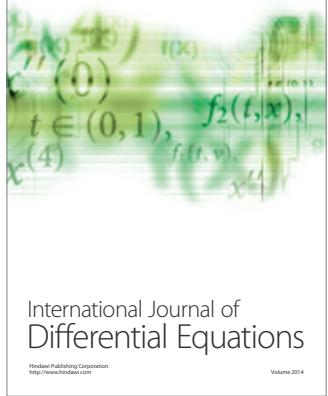
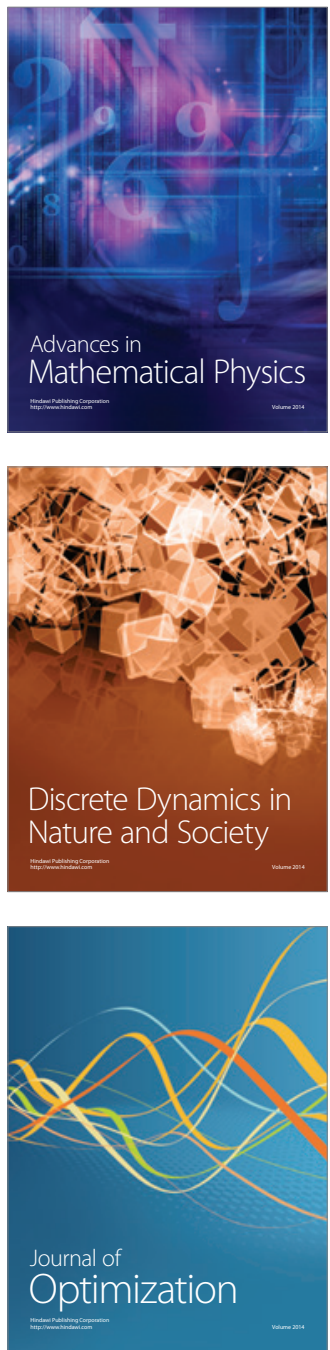